\title{
DEVELOPMENT AND CHARACTERIZATION OF PIPER RETROFRACTUM EXTRACT LOADED MUCOADHESIVE NANOSTRUCTURED LIPID CARRIERS FOR TOPICAL ORAL DRUG DELIVERY
}

\author{
KAVEE SRICHAIVATANA ${ }^{\mathrm{a}, \mathrm{b}}$, ANAN OUNAROON ${ }^{\mathrm{a}}$, WAREE TIYABOONCHAI ${ }^{\mathrm{a}, \mathrm{b}, \mathrm{c}^{*}}$
}

aFaculty of Pharmaceutical Sciences, Naresuan University, Phitsanulok, Thailand, bThe Center of Excellence for Innovation in Chemistry (PERCH-CIC), Commission on High Education, Ministry of Education, Thailand, ${ }^{c}$ The Center of Excellence in Medical Biotechnology, Naresuan University, Phitsanulok, Thailand

Email: wareet@nu.ac.th

Received: 16 May 2017 Revised and Accepted: 13 Jul 2017

\section{ABSTRACT}

Objective: To develop and characterize Piper retrofractum extract loaded nanostructured lipid carriers (PRE loaded NLCs) for topical oral cavity administration to enhance bioavailability and stability of piperine.

Methods: PRE loaded NLCs were prepared with a hot high-pressure homogenization technique followed by coating the particle surface with mucoadhesive polymers; polyethene glycol 400 (PEG) and polyvinyl alcohol (PVA). The physicochemical properties in terms of particle size, polydispersity index, zeta potential, drug entrapment efficiency, in vitro drug release profile and antimicrobial activities were examined. In vitro, mucoadhesion studies were assessed by the wash-off test. The physicochemical stabilities of mouth spray containing PRE loaded NLCs were investigated by kept at room temperature and $4{ }^{\circ} \mathrm{C}$ for 6 mo.

Results: The PRE loaded NLCs showed spherical shape with a mean particle size of $\sim 100-120 \mathrm{~nm}$ and zeta potential of $\sim-24 \mathrm{mV}$. Up to $90 \%$ drug entrapment efficiency was achieved. PEG-NLCs and PVA-NLCs showed a strong interaction with porcine buccal mucosa than uncoated-NLCs. All PRE loaded NLCs formulations revealed fast release characteristics and effective against Streptococcus mutans and S. sanguinis. The mouth spray containing PRE loaded NLCs showed good physical stability without particle aggregation. In addition, the chemical stability of piperine in NLCs was significantly improved during storage at both storage conditions compared to its solution form.

Conclusion: The developed PRE loaded polymer coated-NLCs showed high potential to use as a local drug delivery system for reducing the bacterial growth in the oral cavity.

Keywords: Nanostructured lipid carriers, Mucoadhesive, Piper retrofractum, Piperine

(C) 2017 The Authors. Published by Innovare Academic Sciences Pvt Ltd. This is an open access article under the CC BY license (http://creativecommons.org/licenses/by/4.0/) DOI: http://dx.doi.org/10.22159/ijpps.2017v9i9.19382

\section{INTRODUCTION}

Oral biofilm is a progressive and dynamic process of bacterial adhesion and colonization on the tooth and oral cavity surface. Several reports identify Streptococcus mutans and S. sanguinis as the main contributor in biofilm formation. These bacteria adhere well to the tooth surface and produce a high amount of acid as well as extracellular polysaccharides (sticky glucan) resulting in demineralization of the enamel and further dental plaque formation that can lead to dental caries and periodontitis [1-3]. Thus, in order to prevent these common oral mouth infectious diseases, several methods have been applied to remove bacteria in oral cavities and prevent bacterial recolonization by mechanical cleansing and/or the application of antiseptic agents. Unfortunately, the long term use of antiseptic agents (such as chlorhexidine and hydrogen peroxide) can lead to undesirable side effects such as alterations in taste sensation, burning sensation, and teeth staining [4-6].

This has resulted in a move away from using antiseptic agents towards the use of botanical extracts which possess antimicrobial activities with low side effects. One botanical extract of particular interest in our study was Piper retrofractum fruit extract (PRE), a rich source of alkaloids, especially piperine. Several studies report that piperine is effective against microorganisms including $S$. mutans $[7,8]$. However, the most common oral hygiene products are usually marketed in the solution form. This dosage form does not ensure an adequate drug concentration at the infected area because of the rapid clearance of active substance leading to low therapeutic efficiency. In order to maintain a sufficient therapeutic level, this formulation requires a frequent application which leads to poor patient compliance and increases local side effects due to the high concentration of the drug on the application site [9]. These limitations can be overcome with nanostructured lipid carriers
(NLCs). NLCs improve therapeutic efficiency of the drug by prolonging the drug retention time on the buccal mucosa tissue [10], and by providing controlled drug release near the disease area.

Of interest also are mucoadhesive polymers which have been extensively used as a surface modifier of particulate drug carriers to enhance the bioavailability of the drugs by further intimate contact with mucus layer, which prolong the residence time as well as localizing the drug to a specific region [9]. Polyethylene glycol 400 (PEG) and polyvinyl alcohol (PVA) are widely used as mucoadhesive polymers. They are hydrophilic, biodegradable, less toxicity and presented with a hydroxyl group that could interact with the oligosaccharide chain of mucin [12-14].

Accordingly, this study aimed to develop and characterize PRE loaded uncoated-NLCs and polymer coated-NLCs. Their physicochemical properties in terms of particle size, zeta potential, drug entrapment efficiency, in vitro drug release profile, and mucoadhesiveness to the porcine buccal mucosa were examined. Their antimicrobial activities were also determined. In addition, the physicochemical stabilities of mouth spray containing PRE loaded NLCs were investigated.

\section{MATERIALS AND METHODS}

\section{Materials}

Brain heart infusion (BHI) was purchased from Himedia Laboratories Pvt. Ltd. (Adjani, India). Glyceryl monostearate (GMS), Lexol® GT-865 (medium chain triglyceride, MCT), menthol, myristic acid, poloxamer 407, polyethylene glycol 400 (PEG), and sodium benzoate were purchased from Namsiang Trading Co., Ltd. (Bangkok, Thailand). Polyvinyl alcohol (PVA) was purchased from Ajax Finechem Pty Ltd. (Seven Hills, Australia). Porcine buccal 
tissues were obtained from Bangrakam slaughter house (Phitsanulok, Thailand). Potassium dihydrogen phosphate (Lot No. 070M0019) and standard piperine $(>99 \%$ piperine) (Lot No. U13423V) were purchased from Sigma-Aldrich Co. (Missouri, USA). $S$. mutans (ATCC25175T) and S. sanguinis (ATCC10556) were obtained from the National Institute of health, Department of Medical Sciences (Bangkok, Thailand). Span 20 was obtained from Merck (Darmstadt, Germany). Tween 80 was purchased from S. Tong Chemical (Bangkok, Thailand). Triphenyl tetrazolium chloride (TTC) was purchased from AppliChem GmbH ITW Company (Darmstadt, Germany). All solvents were of analytical grade.

\section{Methods}

Preparation of Piper retrofractum fruit extract (PRE) and piperine content analysis

Dried fruits of $P$. retrofractum, obtained from Manora Dispensary drug store, Phitsanulok, Thailand, were ground into powder and macerated with $95 \%$ ethanol at ambient temperature for $3 \mathrm{~d}$. The extract was filtered and evaporated under reducing pressure condition. The percentage of yield was calculated (Equation 1). The amount of piperine in the extract was analyzed using highperformance liquid chromatography (HPLC). An HPLC system (Shimadzu, Kyoto, Japan) equipped with a reverse-phase C18 column (USP, 4.6 x $150 \mathrm{~mm}$ i.d., $5 \mu \mathrm{m}$, Vertical ${ }^{\circledR}$ Thailand) was used. The mobile phase consisted of acetonitrile and $25 \mathrm{mmol}$ potassium dihydrogen phosphate $65: 35)$. The flow rate was at $1.0 \mathrm{ml} / \mathrm{min}$ and detection wavelength was $340 \mathrm{~nm}$. All data were processed with the LC solution ${ }^{\circledR}$ software (Shimadzu, Kyoto, Japan) [15].

$$
\% \text { yield }=\frac{\text { Dried crude extract }(\mathrm{g})}{\text { Dried powder of long pepper fruit }(\mathrm{g})} \times 100 \text { (1) }
$$

\section{Preparation of PRE-loaded mucoadhesive NLCs}

PRE-loaded uncoated-NLCs were prepared with a hot high-pressure homogenization technique using Microfluidizer (Microfluidics Corporation, Massachusetts, USA). The oil phase was composed of myristic acid, GMS, Lexol® GT-865, and Span 20. The water phase was composed of PRE, Tween 80, propylene glycol, ethanol, and deionized water (DI water). The oil phase and water phase were separately heated to $80^{\circ} \mathrm{C}$. The water phase was then poured into the oil phase and homogenized using a high shear homogenizer (IKA, Staufen, Germany) at $8000 \mathrm{rpm}$ for $1 \mathrm{~min}$. The resulting hot preemulsion was processed through a high-pressure homogenizer (HPH) at 500 bar for 3 cycles, then, cooled down to ambient temperature to obtain PRE loaded NLCs dispersion. Finally, the prepared uncoated-NLCs were washed three times with DI water using an ultrafiltration cell fitted with an MWCO $100 \mathrm{kDa}$ membrane (Millipore, Massachusetts, USA).

Separately from the uncoated-NLCs, 2 formulations of polymer coated-NLCs; PEG-NLCs, and PVA-NLCs were also fabricated by an adsorption technique. PEG or PVA solution $(1 \% \mathrm{w} / \mathrm{v})$ was added to the uncoated-NLC dispersed at a volume ratio of 1:1 and stirred at $500 \mathrm{rpm}$ for $30 \mathrm{~min}$ (WiseStir, Wertheim, Germany). The NLCs were then washed with DI water as described above.

\section{Physicochemical properties of PRE loaded NLCs}

\section{Morphology}

The morphology of the PRE loaded NLCs was examined using a transmission electron microscope (TEM, Philips, Oregon, USA). An aliquot of the PRE loaded NLCs was diluted in $10 \mathrm{ml}$ of DI water, and a $20 \mu \mathrm{l} \mathrm{sample} \mathrm{was} \mathrm{directly} \mathrm{deposited} \mathrm{on} \mathrm{a} \mathrm{carbon-coated} 300$ mesh copper grid and stained with $10 \mu \mathrm{l}$ of uranyl acetate solution $(0.5 \%$ $\mathrm{w} / \mathrm{v}$ ). Excessive solution was removed by Whatman no. 1 filter paper and allowed to air-dry. The specimens were kept in a desiccator until required.

\section{Mean particle size and polydispersity index}

The mean particle size and polydispersity index of the NLCs dispersion were characterized by dynamic light scattering (DLS) employing a ZetaPALS ${ }^{\circledR}$ analyzer (Brookhaven Instruments Corporation, New York, USA), which was equipped with a $35 \mathrm{~mW}$ helium-neon laser diode operating at $632.8 \mathrm{~nm}$ and a BI-200SM
Goniometer connected to a BI-9010AT digital correlator. All samples were diluted with DI water. The particle size of each sample was measured at $25^{\circ} \mathrm{C}$ at a detection angle of $90^{\circ}$ and run for ten times of measurement. The particle size analysis data were evaluated using the hydrodynamic diameter and the measurement was performed in triplicate.

\section{Zeta potential}

Zeta potential was determined by phase analysis light scattering with a ZetaPALS ${ }^{\circledast}$ analyzer. The measurement was performed at $14.8^{\circ}$ to the incident light. All samples were diluted with DI water in a volume ratio of 1:3 in $10 \mathrm{~mm}$ diameter cell. Data were collected for 10 cycles and each sample was determined in triplicate. The zeta potential was calculated from the electrophoresis mobility based on the Smoluchowski equation included in the software of the system.

\section{Piperine incorporation efficiency}

The amount of piperine incorporated in the NLCs was determined by the centrifugation method. Piperine was extracted from the particles by ethanol with sonication for $1 \mathrm{~min}$ at room temperature and then centrifuged at 14,000 rpm for $30 \mathrm{~min}$. The amount of piperine in the supernatant was analyzed by HPLC as described above. Each sample was determined in triplicate. The percentage of piperine incorporated in the NLCs was calculated by the following equation 2 .

$$
\text { Piperine incorporation efficiency }(\%)=\left(\frac{\text { Piperine }_{\text {tested }}}{\text { Piperine }_{\text {initial }}}\right) \times 100(2)
$$

\section{Evaluation of mucoadhesive properties}

The mucoadhesive properties of the PRE loaded NLCs was investigated by the in vitro wash-off method using porcine buccal mucosa, obtained from a local slaughterhouse. The buccal mucosa tissue was separated from the underlying connective tissue with tweezers and surgical scissors, and kept in cold phosphate-buffered saline $(\mathrm{pH}=7)$ until used $(<8 \mathrm{~h})$. A piece of porcine buccal tissue $\left(1 \mathrm{~cm}^{2}\right)$ was mounted on a glass slide with glue. Then $25 \mu \mathrm{l}$ of the PRE loaded NLCs (containing piperine $35 \mu \mathrm{g}$ ) was spread on the porcine buccal mucosa and allowed to equilibrate for $2 \mathrm{~min}$. The tissue was then exposed to a continuous flow of artificial saliva at $37^{\circ} \mathrm{C}$, at a flow rate of $0.5 \mathrm{ml} / \mathrm{min}$ with a peristaltic pump (Cole-Parmer GmbH, Wertheim, Germany). A $6 \mathrm{ml}$ sample of the flushed artificial saliva was taken at predetermined time intervals of 15 , 30, 60, 120 and $180 \mathrm{~min}$. Finally, the piperine in the flushed artificial saliva was extracted using $2 \mathrm{ml}$ of benzene and the benzene was further eliminated with a speed vacuum concentrator (Labconco Corporation, Missouri, USA) at $40^{\circ} \mathrm{C}$ for $50 \mathrm{~min}$. The dried residue was re-dissolved in $500 \mu \mathrm{l}$ of mobile phase and the amount of piperine was determined by HPLC. The percentage of piperine retained on the tissue was calculated by following equation 3 .

$$
\text { Remained piperine }(\%)=100-\left(\frac{\text { piperine }_{\text {washed off }} \times 100}{\text { piperine }_{\text {initial }}}\right)(3)
$$

Where: piperine $_{\text {washed off }}=$ the amount of piperine that flushed by artificial saliva.

piperine $_{\text {initial }}=$ initial amount of piperine in PRE loaded NLCs

\section{In vitro drug release studies}

In vitro drug release of the PRE loaded NLCs was studied by the shake-flask method. Since piperine compound has a poor aqueous solubility, the artificial saliva containing $0.5 \%(\mathrm{v} / \mathrm{v})$ of Tween 80 was used as a dissolution medium to provide the sink condition. The medium was allowed to equilibrate in an orbital shaker at $37 \pm 0.5^{\circ} \mathrm{C}$ for $30 \mathrm{~min}$ before use. A $100 \mu \mathrm{l}$ of PRE loaded NLCs, contained piperine $125 \mu \mathrm{g}$, was added into $7 \mathrm{ml}$ of medium and constantly shaken at $100 \mathrm{rpm}, 37 \pm 0.5^{\circ} \mathrm{C}$. An aliquot of $300 \mu \mathrm{l}$ of the sample was taken at time intervals of $5,10,15,30,60,120,180$, and $360 \mathrm{~min}$. The selected samples were then centrifuged at $18,000 \mathrm{rpm}$ for 30 min and the supernatant was assayed for the amount of piperine released using HPLC as described above.

\section{Antimicrobial activity of PRE and PRE loaded NLCs}

The antibacterial activities of the tested samples were investigated against $S$. mutans and S. sanguinis. The broth microdilution method 
was used to determine the minimum inhibitory concentration (MIC). A stock solution of PRE was prepared by dissolving PRE in BHI broth composed of $0.25 \%(\mathrm{w} / \mathrm{v})$ of Tween 80 and $5 \%(\mathrm{w} / \mathrm{v}$ ) of dimethyl sulfoxide (DMSO). Then two-fold serial dilution of the PRE stock solution was performed in a 96-well plate with BHI to obtain a piperine concentration range from 1000 to $0.48 \mu \mathrm{g} / \mathrm{ml}$. An aliquot of $20 \mu \mathrm{l}$ of bacterial inoculum was added, $10^{6} \mathrm{CFU} / \mathrm{ml}$, into each well and incubated at $37{ }^{\circ} \mathrm{C}$ for $24 \mathrm{~h}$, under anaerobic conditions. The bacterial growth was detected by adding $20 \mu \mathrm{l}$ of TTC $(0.2 \%(\mathrm{w} / \mathrm{v}))$ in each sample well and incubated at $37^{\circ} \mathrm{C}$ for $1 \mathrm{~h}$. The development of red color indicates bacterial growth, and the absence of the color means inhibition of bacterial growth. The positive control (chlorhexidine) and solvent control were also tested in the same way. All NLC formulations were also studied for antibacterial activity similar to the tests for free PRE mentioned above. In addition, the blank NLCs was employed as a control to ensure that the NLC had no effect on bacterial growth. All of the MIC determinations were performed in triplicate.

\section{Preparation of mouth spray containing PRE loaded NLCs}

Mouth sprays were prepared by the beaker method. The water phase composed of sodium benzoate, sodium saccharin, menthol, poloxamer 407, propylene glycol, and DI water. All ingredients were mixed by a magnetic stirrer until homogenous. Spearmint oil and peppermint oil were then added and stirred at $500 \mathrm{rpm}$ for $30 \mathrm{~min}$. Finally, the PRE loaded NLC dispersions were added and mixed to obtain $0.1 \%(\mathrm{w} / \mathrm{w})$ piperine.

\section{Physicochemical stability of NLCs in mouth spray solution}

The mouth spray containing PRE loaded NLCs were stored at room temperature and $4{ }^{\circ} \mathrm{C}$ in a well closed container and protected from light. An aliquot of each sample was taken at intervals of 1, 3, and 6 mo to investigate the mean particle size, zeta potential, and the piperine remaining. Each sample was carried out in triplicate. A mouth spray containing PRE was used as a control. This formulation was obtained by simply replacing the PRE loaded NLCs with PRE.

\section{Statistical analysis}

The results were expressed as mean \pm standard deviation (SD). Differences between the groups were compared by one-way ANOVA followed by Tukey's post hoc test. The results were considered to be statistically significant at $* \mathrm{P}<0.05$.

\section{RESULTS}

\section{Preparation of PRE and piperine content analysis}

Preparation of PRE using the method described above produced the extract with the yield of $16.32 \pm 0.48 \%(\mathrm{w} / \mathrm{w})$ compared to the dried powder weight. The appearance of the extract was a dark viscous liquid. Based on HPLC analysis, piperine was found as the major compound in PRE with $20.94 \pm 2.46 \%$ (w/w).

\section{Physicochemical properties of PRE loaded mucoadhesive NLCs}

The mean particle size of the unloaded NLCs was in the range of 70$90 \mathrm{~nm}$ with a polydispersity index of $<0.3$ indicating a narrow size distribution, and exhibited negatively charged with a zeta potential of $\sim-24 \mathrm{mV}$ (table 1). The entrapment of the piperine produced a marginal effect on the mean particle size. Piperine loading led to an increase in the mean particle size to $100-120 \mathrm{~nm}$ but did not affect the zeta potential and polydispersity index. Furthermore, the mean size of PRE loaded NLCs (uncoated-NLCs) was increased from 100 $\mathrm{nm}$ to $\sim 120 \mathrm{~nm}$ after coating with mucoadhesive polymers. In addition, the piperine incorporation efficiency was found to be higher than $90 \%$.

Table 1: Physicochemical characteristics of blank NLCs and PRE loaded NLCs.

\begin{tabular}{|c|c|c|c|c|c|c|c|}
\hline \multirow[t]{2}{*}{ Formulations } & \multicolumn{3}{|l|}{ Blank NLCs } & \multicolumn{4}{|c|}{ PRE loaded NLCs } \\
\hline & $\begin{array}{l}\text { Mean size } \\
(\mathrm{nm}) \pm \mathrm{SD}\end{array}$ & $\mathrm{PI} \pm \mathrm{SD}$ & $\begin{array}{l}\mathrm{ZP} \\
(\mathrm{mV}) \pm \mathrm{SD}\end{array}$ & $\begin{array}{l}\text { Mean size } \\
(\mathrm{nm}) \pm \mathrm{SD}\end{array}$ & PI \pm SD & $\begin{array}{l}\mathrm{ZP} \\
(\mathrm{mV}) \pm \mathrm{SD}\end{array}$ & $\% E E \pm S D$ \\
\hline Uncoated-NLCs & $72 \pm 4$ & $0.17 \pm 0.02$ & $-25.3 \pm 1.6$ & $104 \pm 4$ & $0.13 \pm 0.04$ & $-24.5 \pm 2.5$ & $97 \pm 2$ \\
\hline PEG-NLCs & $92 \pm 3$ & $0.16 \pm 0.02$ & $-25.0 \pm 1.7$ & $125 \pm 4$ & $0.17 \pm 0.03$ & $-25.0 \pm 2.6$ & $95 \pm 1$ \\
\hline PVA-NLCs & $92 \pm 4$ & $0.19 \pm 0.02$ & $-24.2 \pm 2.4$ & $128 \pm 6$ & $0.17 \pm 0.01$ & $-25.4 \pm 2.1$ & $95 \pm 3$ \\
\hline
\end{tabular}

PI: polydispersity index; ZP: zeta potential; EE: entrapment efficiency; SD: standard deviation for $\mathrm{n}=3$.

TEM micrographs revealed that PRE loaded uncoated-and polymer coated-NLCs were spherical in shape and uniformly distributed. In the case of PEG-NLCs and PVA-NLCs, as shown in fig. 1B and 1C, respectively, the light gray appeared on the outer layer of the particle was attributed to the polymer coating on the outer surface particles. The particle size of polymer coated-NLCs $(\sim 120 \mathrm{~nm})$ was found to be slightly larger than uncoated-NLCs $(\sim 100 \mathrm{~nm})$. The result from TEM was similar to that obtained by DLS.

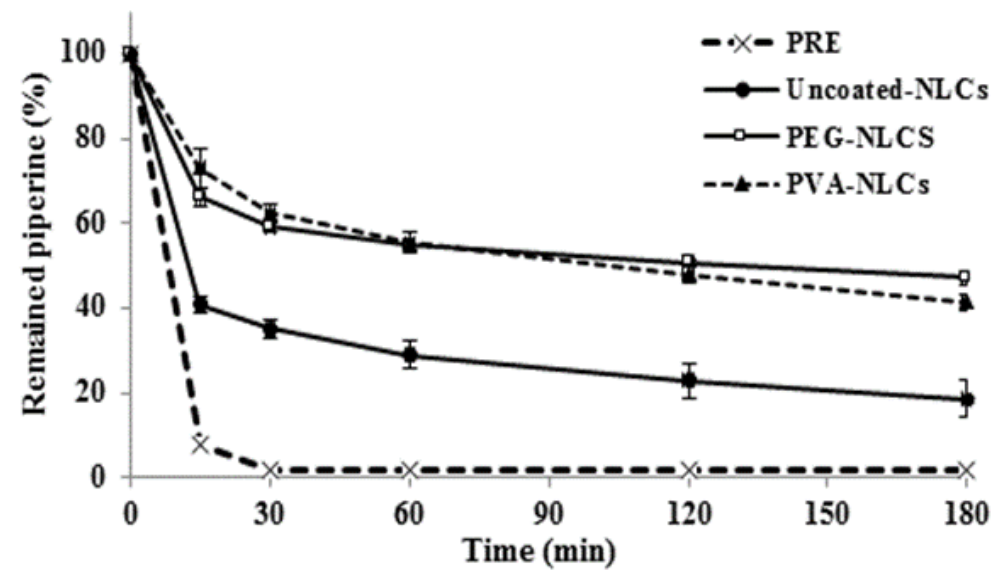

Fig. 2: Mucoadhesive properties of (--×-) PRE, and PRE loaded (-๑) uncoated-NLCs, ( $\square-)$ PEG-NLCs, and (--^-- ) PVA-NLCs. Error bars indicate SD for $\mathbf{n}=3$. 


\section{Evaluation of mucoadhesive properties}

Instillation of free PRE on the buccal mucosa resulted in significant loss of piperine after 15 min of fluid flow (>90\%), and only $2 \%$ of piperine remained on buccal mucosa after $180 \mathrm{~min}$ (fig. 2). However, on the instillation of uncoated-NLCs and polymer coated-NLCs exhibited superior retention to the porcine mucosa.
After 15 min of fluid flow, uncoated-NLCs showed 40\% piperine remaining on the buccal mucosa and after 180 min only $\sim 18 \%$ remained. The surface modification with PEG and PVA showed greater mucoadhesiveness than uncoated-NLCs. Over $66 \%$ of piperine remained on buccal mucosa after 15 min and $~ 47 \%$ of piperine remained after 180 min of fluid flow.

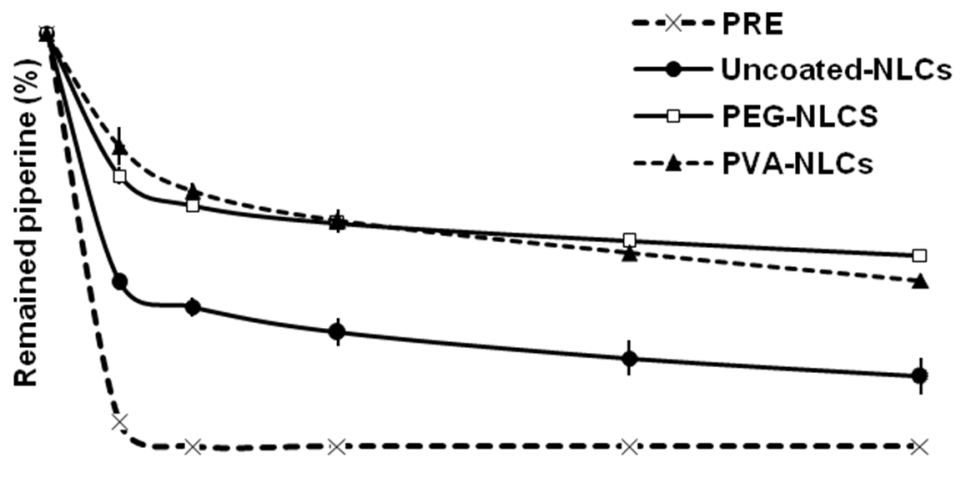

Time (min)

Fig. 2: Mucoadhesive properties of (-- $\times--)$ PRE, and PRE loaded ( $\longrightarrow-)$ uncoated-NLCs, $(-\square-)$ PEG-NLCs, and (--A--) PVANLCs. Error bars indicate SD for $n=3$

\section{In vitro drug release studies}

Artificial saliva containing Tween 80 was used as a dissolution medium to provide sink condition because of the piperine's low water solubility. The solubility of piperine in dissolution medium was $\sim 350 \mu \mathrm{g} / \mathrm{ml}$. All NLC formulations showed a fast release profile with a burst release in the first $5 \mathrm{~min}$ (fig. 3). The uncoated-NLCs exhibited $\sim 40 \%$ piperine released in the first $5 \mathrm{~min}$ and $\sim 60 \%$ in
60 min, whereas the PEG-NLCs and PVA-NLCs showed higher piperine released of $\sim 55 \%$ and $\sim 70 \%$ after 5 and $15 \mathrm{~min}$ respectively. Obviously, PEG-NLCs and PVA-NLCs showed the higher initial drug released than uncoated-NLCs $\left({ }^{*} \mathrm{P}<0.05\right)$.

The drug release pattern suggested that most of the piperine, $70 \%$, was localized at or near the particle surface with $\sim 30 \%$ of the piperine entrapped inside the NLCs lipid matrix.

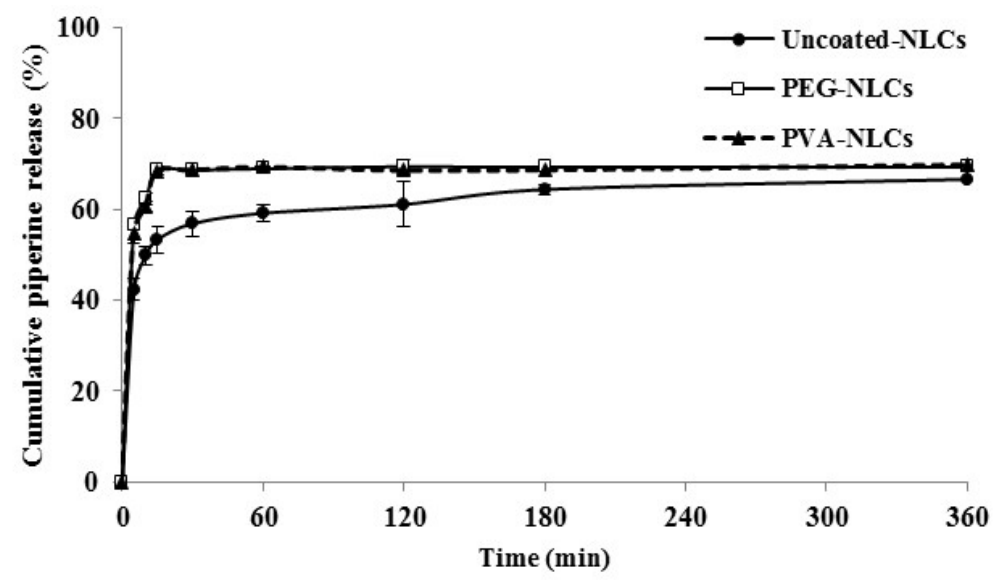

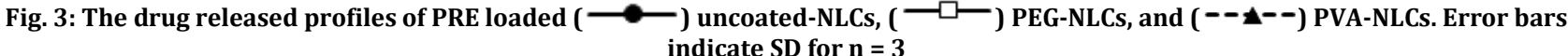

\section{Antimicrobial activity of PRE and PRE loaded NLCs}

The free PRE and PRE loaded NLCs were active against the tested bacteria (table 2). The MIC value of free PRE, based on the piperine content, against $S$. mutans and S. sanguinis were 125 and $250 \mu \mathrm{g} / \mathrm{ml}$, respectively, while all NLC formulations showed the higher MIC value for all tested bacteria at $1000 \mu \mathrm{g} / \mathrm{ml}$ (table 2). This result was attributed to the tightly entrapped of piperine in particles and incomplete release into the culture medium. Nevertheless, the blank NLCs and solvent showed no effect against the tested bacteria (data not show). Therefore, the antibacterial activity of PRE loaded NLCs was resulted from piperine.

\section{Physicochemical stability of NLCs in mouth spray solution}

After 6 mo storage at room temperature, the mean particle size and zeta potential showed no significant difference compared with a freshly preparation (table 3 ). The mean particle size was practically unchanged at $\sim 100-120 \mathrm{~nm}$. On the other hand, the mean particle size of all NLC formulations tends to be of a larger size after being stored at $4{ }^{\circ} \mathrm{C}$ for 1,3 and 6 mo suggesting an aggregation process of the nanoparticle. In addition, TEM micrographs revealed that the particle shape changed from spherical to palette after storage at $4{ }^{\circ} \mathrm{C}$ (table 4). Nevertheless, the zeta potential was not changed significantly under both storage conditions. 
Table 2: Minimum inhibitory concentration (MIC) of PRE, PRE loaded NLCs, and chlorhexidine against oral pathogens determined by broth microdilution method

\begin{tabular}{lll}
\hline & MIC $(\mu \mathrm{g} / \mathrm{ml})$ & \\
\cline { 2 - 3 } & S. mutans & S. sanguinis \\
\hline PRE & 125 & 250 \\
Uncoated-NLCs & 1000 & 1000 \\
PEG-NLCs & 1000 & 1000 \\
PVA-NLCs & 1000 & 1000 \\
Chlorhexidine & 7.8 & 7.8 \\
\hline
\end{tabular}

Note: the MIC value of PRE and PRE loaded NLCs were based on the concentration of piperine.

Table 3: The mean particle size and zeta potential of PRE loaded NLCs in mouth spray after storage for 6 mo at room temperature and $4{ }^{\circ} \mathrm{C}$ in the absence of light

\begin{tabular}{|c|c|c|c|c|c|c|}
\hline \multirow[t]{2}{*}{ Storage temperature } & \multicolumn{3}{|c|}{ Mean size $(\mathrm{nm}) \pm \mathrm{SD}$} & \multicolumn{3}{|c|}{ Zeta potential $(\mathrm{mV}) \pm \mathrm{SD}$} \\
\hline & Uncoated-NLCs & PEG-NLCs & PVA-NLCs & Uncoated-NLCs & PEG-NLCS & PVA-NLCs \\
\hline Initial & $109 \pm 0$ & $117 \pm 0$ & $112 \pm 4$ & $-25.3 \pm 0.8$ & $-24.9 \pm 2.6$ & $-25.4 \pm 2.0$ \\
\hline \multicolumn{7}{|l|}{ After storage at RT } \\
\hline $1 \mathrm{mo}$ & $112 \pm 11$ & $122 \pm 4$ & $117 \pm 2$ & $-25.8 \pm 1.8$ & $-24.0 \pm 2.9$ & $-25.5 \pm 0.5$ \\
\hline $3 \mathrm{mo}$ & $106 \pm 6$ & $117 \pm 3$ & $117 \pm 2$ & $-23.7 \pm 1.9$ & $-23.3 \pm 0.9$ & $-25.5 \pm 0.5$ \\
\hline $6 \mathrm{mo}$ & $114 \pm 7$ & $123 \pm 5$ & $119 \pm 5$ & $-25.1 \pm 2.7$ & $-23.1 \pm 2.9$ & $-23.9 \pm 3.1$ \\
\hline \multicolumn{7}{|l|}{ After storage at $4{ }^{\circ} \mathrm{C}$} \\
\hline $1 \mathrm{mo}$ & $226 \pm 5$ & $227 \pm 5$ & $246 \pm 3$ & $-24.2 \pm 1.8$ & $-23.6 \pm 2.0$ & $-24.7 \pm 2.3$ \\
\hline $3 \mathrm{mo}$ & $285 \pm 5$ & $294 \pm 4$ & $316 \pm 4$ & $-25.3 \pm 2.1$ & $-23.3 \pm 0.9$ & $-23.9 \pm 3.1$ \\
\hline $6 \mathrm{mo}$ & $282 \pm 2$ & $301 \pm 13$ & $398 \pm 7$ & $-24.2 \pm 1.3$ & $-24.3 \pm 1.9$ & $-23.9 \pm 0.8$ \\
\hline
\end{tabular}

Note: $\mathrm{SD}=$ standard deviation for $\mathrm{n}=3 ; \mathrm{RT}=$ room temperature.

Table 4: Transmission electron micrographs of PRE loaded NLCs after storage at room temperature and $4{ }^{\circ} \mathrm{C}$ for 6 mo

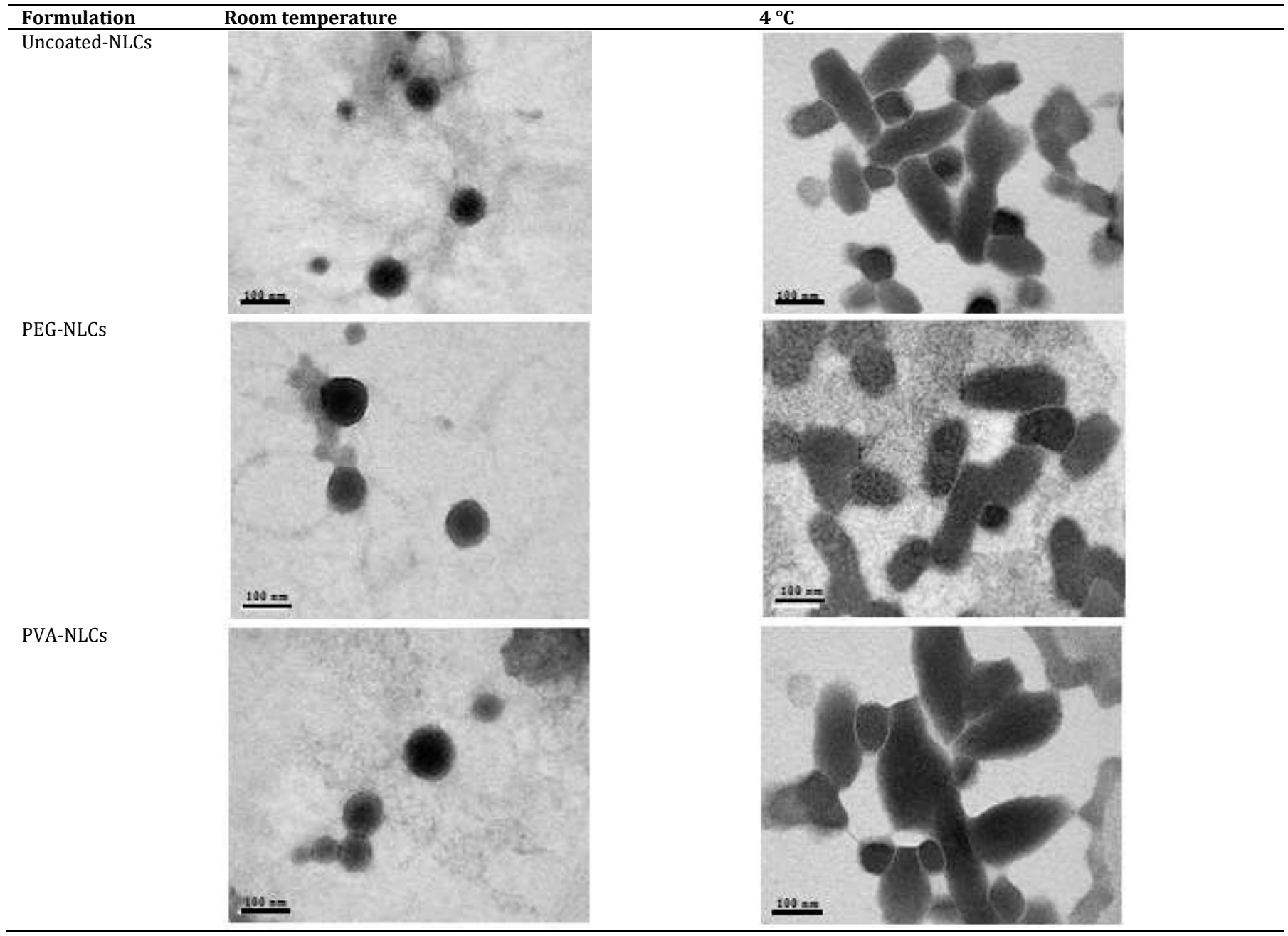


The chemical stability was also investigated in term of piperine remaining. The piperine content of mouth spray formulations significantly decreased in both storage conditions (fig. 4). The mouth spray containing free PRE showed $\sim 77 \%$ piperine remaining after 1 mo storage and further decreased to $\sim 50 \%$ after $6 \mathrm{mo}$ in all storage conditions. On the other hand, the mouth spray containing PRE loaded NLCs showed excellent potential for piperine protection, which increased the percentage of piperine remaining up to $>80 \%$ and $>70 \%$ after 1 and 6 mo storage, respectively, at both room temperature and $4^{\circ} \mathrm{C}$.

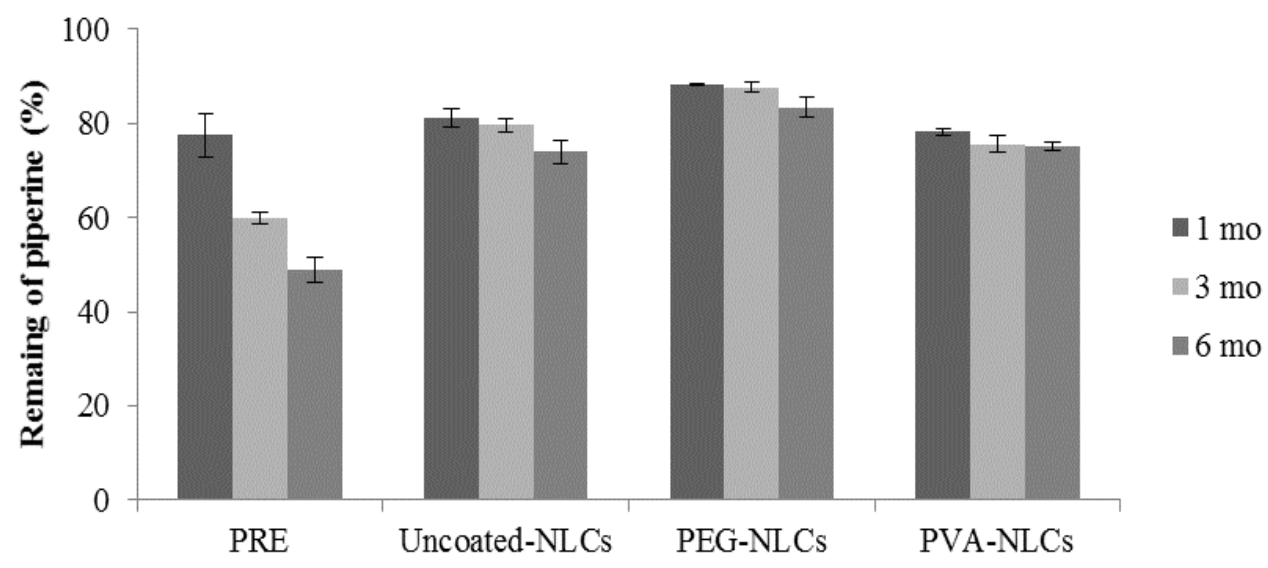

A

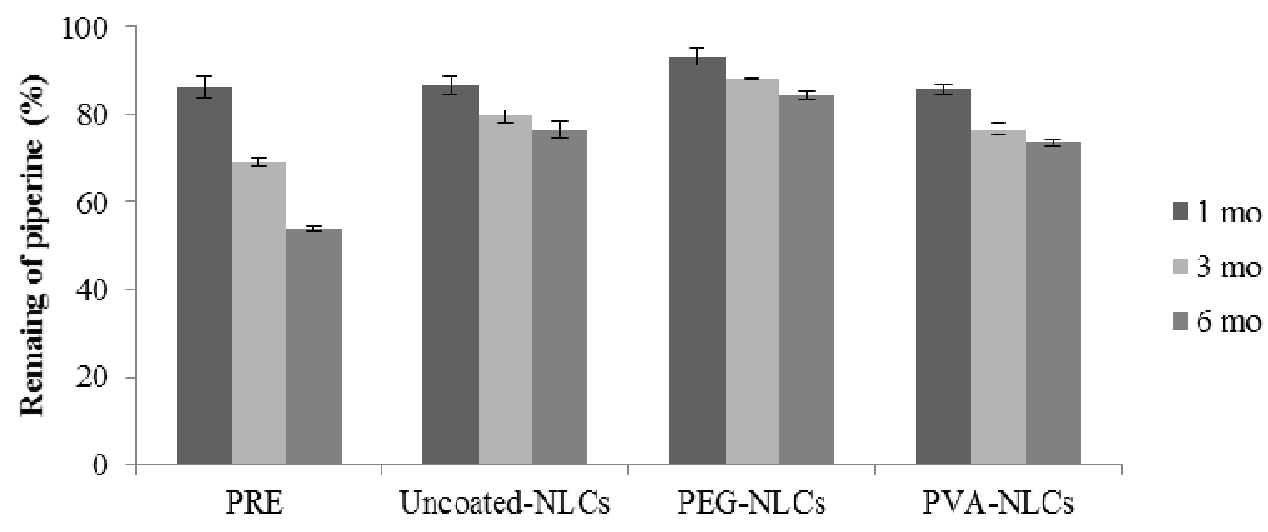

B

Fig. 4: Percentage of piperine remaining after 6 mo storage of PRE and PRE loaded NLCs at (A) room temperature and (B) $4 \stackrel{\circ}{\circ}$. Error bars indicate SD for $\mathrm{n}=3$.

\section{DISCUSSION}

Dental caries and periodontitis are infectious diseases caused by oral pathogens that could be prevented by antibacterial agents. Unfortunately, the chemical antimicrobial agent may lead to undesirable side effects. Hence, the trend has shifted to botanical extracts. However, their therapeutic efficiency is hampered by short residence time from the site of application due to saliva flow and oral muscular functions. Thus, to improve its therapeutic efficacy, NLCs would be a desirable approach. This study demonstrated that mucoadhesive polymer coated-NLCs exhibited promising potential actions for local oral cavity drug administration.

PRE loaded NLCs were successfully prepared by a hot high-pressure homogenization technique. Then, the particle was further coated with mucoadhesive polymers to improve the bioavailability of the drugs by prolonging the residence time on the mucosa. PEG and PVA were believed to attach to the particle surface via hydrogen bonding and acted as a steric stabilizer preventing particle aggregation [16, 17]. The mean particle size of PEG-NLCs and PVA-NLCs was slightly increased, $120 \mathrm{~nm}$, as compared to uncoated-NLCs, $100 \mathrm{~nm}$, suggesting the success of the polymer coating process. Further evidence of polymer coating is consistent with their TEM micrographs that exhibit a light gray layer on the outer layer.

As expected, all NLC formulations exhibited greater adherence to the porcine buccal mucosa as compared to free PRE confirming their mucoadhesive properties. The particle size and the nature of coated polymer are the main parameters affecting mucoadhesive potential. In general, the mucosa tissue is coated with a mucus gel layer composed of glycoprotein fibers mesh that could physically trap small particle sizes of $<200 \mathrm{~nm}[18,19]$. As a consequence, the uncoated-NLCs with mean particle size $\sim 100 \mathrm{~nm}$ could be immobilized within the mucus layer, and thus, avoid elimination by the fluid flow. Furthermore, PEG-NLCs and PVA-NLCs showed a 2fold greater mucosa adhesion than uncoated-NLCs. Their strong mucoadhesive properties were attributed to the PEG and PVA hydrophilic nature with a high hydroxyl content. Upon particle contact with buccal mucosa tissue, their small size of $\sim 120 \mathrm{~nm}$ could facilitate them to diffuse into the mucus layer. At the same time, the polymer chain could diffuse and interpenetrate into the 
mucus gel resulting in the entanglement with mucin, and subsequently, interacting with mucin via hydrogen bonding [12, 20]. Based on these results, PEG-NLCs and PVA-NLCs exhibited a strong mucoadhesion affinity for porcine buccal mucosa. Therefore, the particles could be retained on the buccal mucosa for an extended period of time. This could increase drug concentration at the target site leading to improved therapeutic efficiency of loaded drugs.

In vitro drug release profile of NLCs was determined as it is crucial for an effective local oral drug delivery system. A similar drug release profile was observed for both uncoated-NLCs and polymer coated-NLCs. All formulations illustrated a rapid burst release in the first $5 \mathrm{~min}$. The high initial burst release of piperine was attributed to most of the piperine located at or nearby the particle surface. This phenomenon may be caused by the preparation process. During the heating process, the water solubility of piperine increased, and piperine, in lipid melted droplets were able to diffuse into the water phase. However, in the cooling process, the water solubility of piperine decreased resulting in a re-partition of piperine back into the lipid phase. At the same time, the molten lipid is solidified, thus, the drug could be accessed only in the liquid outer shell and on the particle surface [21]. Interestingly, after $30 \mathrm{~min}$, only $70 \%$ of the piperine was released and remained constant for $360 \mathrm{~min}$. The incomplete drug released may be explained by a slow diffusion of piperine from oil nano compartment into the solid lipid matrix before releasing into the medium. Due to the high amount of MCT, $57 \%$ of the total lipid phase, the oil was separated as fine droplets being incorporated into the solid lipid matrix during the cooling process leading to the formation of multiple type NLCs [21].

PEG-NLCs and PVA-NLCs showed higher amounts of piperine released than uncoated-NLCs after the first $5 \mathrm{~min},{ }^{*} \mathrm{P}<0.05$. It could be postulated that the mucoadhesive polymers enhance drug release by two mechanisms. First, the mucoadhesive polymer could interact with piperine by hydrogen bonding and is localized at the particle surface thereby being responsible for the initial burst release. Second, the water-soluble property of these polymers might further help the piperine leach out from the particle surface to the dissolution medium [22, 23]. The initial burst release of PRE loaded NLCs could be considered as an advantage to provide a sufficient therapeutic dose for inhibiting bacterial growth in a timely manner. Interestingly, in vitro antibacterial studies have revealed that all NLC formulations are effective against both tested bacteria. However, the PRE loaded NLCs showed 8-fold higher MIC value against $S$. mutans, and 4-fold higher value against $S$. sanguinis than the free PRE. The blank NLCs showed no antibacterial activity indicating that the piperine released from NLCs is responsible for antibacterial activity. However, the observed higher MIC value of the NLCs suggests that the incomplete release of piperine from the particles is due to the culture medium not providing sink conditions whereas the dissolution medium did. Nevertheless, this observation could be clinically useful because of the intrinsically low water solubility of piperine which could provide a sustained release effect. In addition, all NLC formulations demonstrated strong mucoadhesive properties to the buccal mucosa tissue. This property could reduce the biofilm formation by a competitive adhesion between the nanoparticles and the bacteria to the buccal mucosa tissue. Ahn et al. showed that the silver nanoparticles can reduce adhesion of the $S$. mutans on experimental composite adhesives [24]. Additionally, Chávez de Paz et al. showed that chitosan nanoparticles were capable of reducing the adhesion and penetration of $S$. mutans through the biofilm structure at neutral $\mathrm{pH}$ [25].

The physical stability of NLCs is the main concern due to their instability in the liquid medium. The developed mouth spray containing PRE loaded NLCs showed good physical stability with no evidence of particle aggregation or precipitation when stored at room temperature. Surprisingly, the mean particle size progressively increased with time and the particle shape transformed to be platelet-like when kept at $4{ }^{\circ} \mathrm{C}$. This phenomenon can be described by polymorphic transformation of the inner lipid matrix of NLCs. Jenning et al. reported that the low temperature could induce the polymorphic transition of MCT from the amorphous form ( $\alpha$-form) to crystallized form ( $\beta$-form), leading to the transformation of spherical to platelet shaped particles [26, 27]. In addition, Helgason et al. stated that the surfactants required to cover and stabilize the newly created interfaces may not be able to diffuse rapidly enough to adsorb at the newly formed lipid crystalline interfaces, leading to increased mean particle size due to flocculation [28].

The chemical stability of piperine in nanoparticles is also an important issue. Piperine is susceptible to degradation by oxidation and hydrolysis [29]. As expected, all NLC formulations showed excellent piperine protection, more than its solution form, demonstrating the protective effects of the solid matrix. This improvement of piperine stability is attributed to the lipid matrix preventing or reducing the diffusion of the aqueous medium into nanoparticles. These results indicate that NLCs improve piperine stability. However, the amount of piperine incorporated into the NLCs also decreased after 6 mo storage. The degradation of the piperine during storage in the mouth spray formulations was a result of the piperine released from the NLCs into the mouth spray solution. Hence, the addition of an antioxidant into the mouth spray formulation would improve the chemical stability of the piperine.

\section{CONCLUSION}

In conclusion, uncoated-NLCs and polymer coated-NLCs all showed spherical shape with a mean particle of $\sim 100$ to $\sim 120 \mathrm{~nm}$ and zeta potential of $\sim-24 \mathrm{mV}$ with a high entrapment efficiency of $>90 \%$. The developed NLC formulations have greater mucoadhesive properties than the free PRE. In addition, PEG-NLCs and PVA-NLCs showed strong adherence to the porcine buccal mucosa, twice that of the uncoated-NLCs. The ability of PEG-NLCs and PVA-NLCs adhering on the mucosal surface improves the therapeutic efficiency of PRE and/or other drugs by prolonging the residence time of the drug delivery system to the buccal mucosa tissue. In addition, this particulate system is potentially more effective in inhibiting the growth of bacteria in the oral cavity leading to retardation or prevention of biofilm formation. Although showing the rapid drug release pattern, the NLCs is clinically useful because of the intrinsically low water solubility of piperine providing a sustained release effect. In addition, the PRE loaded NLCs showed good effectiveness against $S$. mutans and $S$. sanguinis with an MIC value of $1000 \mu \mathrm{g} / \mathrm{ml}$. Furthermore, the chemical stability of piperine in a mouth spray containing PRE loaded NLCs was significantly improved over free PRE in the mouth spray formulation. As well PRE loaded NLCs showed good physical stability without particle aggregation after 6 mo storage at room temperature. Based on our results, we are confident that developed PRE loaded NLCs is highly advantageous for the prevention of dental caries and periodontitis disease, furthermore, the developed NLCs are a good choice for use in the local oral mucosa drug delivery system.

\section{ACKNOWLEDGEMENT}

The authors gratefully acknowledge Naresuan University and Center of Excellence for Innovation in Chemistry (PERCH-CIC) for the financial support of this work. Special thanks to the Faculty of Pharmaceutical Sciences, Naresuan University for providing the necessary facilities. Many thanks to Mr. Roy Morien of the Naresuan University Language Centre for his editing assistance and advice on English expression in this document.

\section{AUTHORS CONTRIBUTION}

Srichaivatana K makes substantial contributions to acquisition of data, analysis and interpretation of data and drafting the article. Ounaroon A participates in concept, and data analysis and interpretation. Tiyaboonchai $\mathrm{W}$ participates in concept, guidance, critical review, and article revision.

\section{CONFLICTS OF INTERESTS}

All authors have none to declare

\section{REFERENCES}

1. Schmidt JC, Bux M, Filipuzzi-Jenny E, Kulik EM, Waltimo T, Weiger $\mathrm{R}$, et al. Influence of time, toothpaste and saliva in the retention of Streptococcus mutans and Streptococcus sanguinis on different toothbrushes. J Appl Oral Sci 2014;22:152-8. 
2. Spatafora G, Rohrer K, Barnard D, Michalek S. A Streptococcus mutans mutant that synthesize elevated levels of the intracellular polysaccharide is hypercariogenic in vivo. Infect Immun 1995;63:2556-63.

3. Kolenbrander PE, Palmer RJ, Periasamy S, Jakubovics NS. Oral multi species biofilm development and the key role of cell-cell distance. Nat Rev Microbiol 2010;8:471-80.

4. Zanela NL, Bijella MF, Rosa OP. The influence of mouthrinses with antimicrobial solutions on the inhibition of dental plaque and on the levels of mutans streptococci in children. Pesqui Odontol Bras 2002;16:101-6.

5. Eley BM. Antibacterial agents in the control of supragingival plaque-a review. Br Dent J 1999;186:286-96.

6. Tombes MB, Gallucci B. The effects of hydrogen peroxide rinses on the normal oral mucosa. Nurs Res 1993;42:332-7.

7. Dwivedi D, Singh V. Effects of the natural compounds embelin and piperine on the biofilm-producing property of Streptococcus mutans. Afr J Tradit Complementary Altern Med 2016;6:57-61.

8. Aneja KR, Joshi R, Sharma C, Aneja A. Antimicrobial efficacy of fruit extracts of two piper species against selected bacterial and oral fungal pathogens. Braz J Oral Sci 2010;9:421-6.

9. Fang L, Wang L, Yao Y, Zhang J, Wu X, Li X, et al. Micro-and nano-carrier systems: the noninvasive and painless local administration strategies for disease therapy in mucosal tissues. Nanomedicine 2017;13:153-71.

10. Aher SS, Malsane ST, Saudagar RB. Nanosuspension: an overview. Int J Curr Pharm Res 2017;9:19-23.

11. Huang Y, Leobandung W, Foss A, Peppas NA. Molecular aspects of muco-and bioadhesiom: tethered structures and site-specific surfaces. J Controlled Release 2000;65:63-71.

12. Popov A, Enlow E, Bourassa J, Chen H. Mucus-penetrating nanoparticles made with "mucoadhesive" polyvinyl alcohol. Nanomedicine 2016;12:1863-71.

13. Rabanel JM, Hildgen $P$, Banquy $X$. Assessment of PEG on polymeric particles surface, a key step in drug carrier translation. J Controlled Release 2014;185:71-87.

14. Bajad S, Singla AK, Bedi KL. Liquid chromatographic method for determination of piperine in rat plasma: application to pharmacokinetics. J Chromatogr B 2002;776:245-9.

15. Gref R, Domb A, Quellec P, Blunk T, Müller RH, Verbavatz JM, et al. The controlled intravenous delivery of drugs using PEGcoated sterically stabilized nanospheres. Adv Drug Delivery Rev 1995;16:215-33.

16. Acar HY, Garaas RS, Syud F, Bonitatebus P, Kulkarni AM. Superparamagnetic nano particles stabilized by polymerized PEGylated coatings. J Magn Magn Mater 2005;293:1-7.

17. Matsui H, Wagner VE, Hill DB, Schwab UE, Rogers TD, Button B, et al. A physical linkage between cystic fibrosis airway surface dehydration and Pseudomonas aeruginosa biofilms. Proc Natl Acad Sci USA 2006;103:18131-6.

18. Dawson M, Wirtz D, Hanes J. Enhanced viscoelasticity of human cystic fibrotic sputum correlates with increasing micro heterogeneity in particle transport. J Biol Chem 2003;278:50393-401.

19. Yang M, Lai SK, Yu T, Wang YY, Happe C, Zhong W, et al. Nanoparticle penetration of human cervicovaginal mucus: the effect of polyvinyl alcohol. J Controlled Release 2014;192:202-8.

20. Müller RH, Radtke M, Wissing SA. Solid lipid nanoparticles (SLN) and nanostructured lipid carriers (NLC) in cosmetic and dermatological preparations. Adv Drug Delivery Rev 2002;54 Suppl 1:131-55.

21. Makadia HK, Siegel SJ. Poly lactic-co-glycolic acid (PLGA) as biodegradable controlled drug delivery carrier. Polymers (Basel) 2011;3:1377-97.

22. Fontana G, Licciardi M, Mansueto S, Schillaci D, Giammona G. Amoxicillin-loaded poly ethyl cyanoacrylate nanoparticles: influence of PEG coating on the particle size, drug release rate and phagocytic uptake. Biomaterials 2001;22:2857-65.

23. Ahn SJ, Lee SJ, Kook JK, Lim BS. Experimental anti microbial orthodontic adhesives using nanofillers and silver nanoparticles. Dent Mater 2009;25:206-13.

24. Chávez de Paz LE, Resin A, Howard KA, Sutherland DS, Wejse PL. Antimicrobial effect of chitosan nanoparticles on Streptococcus mutans biofilms. Appl Environ Microbiol 2011;77:3892-5.

25. Jenning V, Thünemann AF, Gohla SH. Characterisation of a novel solid lipid nanoparticle carrier system based on binary mixtures of liquid and solid lipids. Int J Pharm 2000;199:167-77.

26. Jenning V, Schäfer-Korting M, Gohla SH. Vitamin loaded solid lipid nanoparticles for topical use: drug release properties. J Controlled Release. 2000;66:115-26.

27. Helgason T, Awad TS, Kristbergsson K, McClements DJ, Weiss J. Influence of polymorphic transformations on gelation of tripalmitin solid lipid nanoparticle suspensions. J Am Oil Chem Soc 2008;85:501-11.

28. Kotte SCB, Dubey PK, Murali PM. Identification and characterization of stress degradation products of piperine and profiling of a black pepper (Piper nigrum L.) extract using LC/Q-TOF-dual ESI-MS. Anal Methods 2014;6:8022-9.

\section{How to cite this article}

- Kavee Srichaivatana, Anan Ounaroon, Waree Tiyaboonchai. Development and characterization of Piper retrofractum extract loaded mucoadhesive nanostructured lipid carriers for topical oral drug delivery. Int J Pharm Pharm Sci 2017;9(9):79-86. 